Proceedings of the Edinburgh Mathematical Society (2006) 49, 735-737 (C)

DOI:10.1017/S0013091505000581 Printed in the United Kingdom

\title{
A COUNTEREXAMPLE TO A CONTINUED FRACTION CONJECTURE
}

\author{
IAN SHORT \\ Department of Mathematics, Logic House, National University of Ireland, Maynooth, \\ Maynooth, Co. Kildare, Ireland (ian.short@nuim.ie)
}

(Received 25 April 2005)

\begin{abstract}
It is known that if $a \in \mathbb{C} \backslash\left(-\infty,-\frac{1}{4}\right]$ and $a_{n} \rightarrow a$ as $n \rightarrow \infty$, then the infinite continued fraction with coefficients $a_{1}, a_{2}, \ldots$ converges. A conjecture has been recorded by Jacobsen et al., taken from the unorganized portions of Ramanujan's notebooks, that if $a \in\left(-\infty,-\frac{1}{4}\right)$ and $a_{n} \rightarrow a$ as $n \rightarrow \infty$, then the continued fraction diverges. Counterexamples to this conjecture for each value of $a$ in $\left(-\infty,-\frac{1}{4}\right)$ are provided. Such counterexamples have already been constructed by Glutsyuk, but the examples given here are significantly shorter and simpler.
\end{abstract}

Keywords: continued fractions; Möbius transformations; iteration; dynamics

2000 Mathematics subject classification: Primary 40A15; 30B70

\section{Introduction}

For each $n \in \mathbb{N}$, let $a_{n}$ be a non-zero complex number and let $t_{n}$ be the Möbius transformation $t_{n}(z)=a_{n} /(1+z)$; then the continued fraction

$$
\boldsymbol{K}\left(a_{n} \mid 1\right)=\frac{a_{1}}{1+\frac{a_{2}}{1+\frac{a_{3}}{1+\cdots}}}
$$

is considered to converge if the sequence with the $n$th term equal to the $n$-fold composition $t_{1} \cdots t_{n}(0)$ converges within the extended complex plane $\mathbb{C}_{\infty}$. We identify the continued fraction (1.1) with the sequence $t_{1}, t_{2}, \ldots$ of Möbius transformations. A problem derived from the private notebooks of Ramanujan is posed in [4, p. 38], which asks whether, for a given complex number $a \neq-\frac{1}{4}$ and a sequence $a_{1}, a_{2}, \ldots$ that converges to $a$, the continued fraction $\boldsymbol{K}\left(a_{n} \mid 1\right)$ diverges if and only if $a \in\left(-\infty,-\frac{1}{4}\right)$. In this paper it is demonstrated that $\boldsymbol{K}\left(a_{n} \mid 1\right)$ may or may not converge if $a \in\left(-\infty,-\frac{1}{4}\right)$, thereby proving the conjecture to be false. Glutsyuk has already provided such examples in [3], but the methods here are significantly shorter and simpler. Our conclusions are summarized in a theorem, whose proof is postponed until $\S 3$. 
Theorem 1.1. If $a \in\left(-\infty,-\frac{1}{4}\right)$, then there are sequences $a_{n}$ of real numbers that converge to a for which $\boldsymbol{K}\left(a_{n} \mid 1\right)$ converges and there are sequences $a_{n}$ of real numbers that converge to a for which $\boldsymbol{K}\left(a_{n} \mid 1\right)$ diverges.

\section{Iteration of a single Möbius transformation}

To understand the dynamics of the sequence $t_{1} \cdots t_{n}$, where $t_{n}(z)=a_{n} /(1+z)$ and $a_{n} \rightarrow a$ as $n \rightarrow \infty$, one must first understand the dynamics of the sequence formed through iterating the Möbius map $t(z)=a /(1+z)$. The theory of iteration of a single Möbius transformation is well known (see, for example, $[\mathbf{1}]$ or $[\mathbf{5}]$ ) and it is independent of continued fractions. We elaborate briefly on this theory.

The conjugacy type of a given Möbius transformation $f(z)=(A z+B) /(C z+D)$ may be determined from the conjugation-invariant quantity $T(f)=(A+D)^{2} /(A D-B C)$ : if $T(f) \in[0,4)$, then $f$ is elliptic; if $T(f)=4$, then $f$ is parabolic; otherwise $f$ is loxodromic. Therefore, $t$ is elliptic if $a \in\left(-\infty,-\frac{1}{4}\right)$, parabolic if $a=-\frac{1}{4}$, and loxodromic otherwise.

If $t$ is loxodromic and $a_{n} \rightarrow a$ as $n \rightarrow \infty$, it follows from the general theory (see [2] or [6]) that $\boldsymbol{K}\left(a_{n} \mid 1\right)$ converges. If $t$ is parabolic and $a_{n} \rightarrow a$ as $n \rightarrow \infty, \boldsymbol{K}\left(a_{n} \mid 1\right)$ may converge or it may diverge, and it is easy to construct examples of both circumstances. This leaves the situation of Theorem 1.1, when $t$ is elliptic. Elliptic maps are by definition conjugate to Möbius maps of the form $z \mapsto \mathrm{e}^{\mathrm{i} \theta} z$, where $\theta \in(0,2 \pi)$, hence $t^{n}(0)$ diverges (since 0 is not a fixed point of $t$ ), that is, $\boldsymbol{K}(a \mid 1)$ diverges. Thus, for one part of Theorem 1.1 we may choose $a_{n}$ to be the constant sequence $a, a, \ldots$ The other part of Theorem 1.1 is proved in $\S 3$.

\section{Proof of Theorem 1.1}

We need a preliminary lemma.

Lemma 3.1. The subset of $\left(-\infty,-\frac{1}{4}\right)$ consisting of those numbers $a \in\left(-\infty,-\frac{1}{4}\right)$ for which $t(z)=a /(1+z)$ is a map of finite order is a dense subset of $\left(-\infty,-\frac{1}{4}\right)$.

Proof. Let $t$ be conjugate to $g(z)=\mathrm{e}^{\mathrm{i} \theta} z, \theta \in(0,2 \pi)$; then

$$
-1 / a=T(t)=T(g)=4 \cos ^{2} \frac{1}{2} \theta .
$$

The maps $t$ and $g$ are of finite order if and only if $\theta$ is a rational multiple of $\pi$, and rational multiples of $\pi$ are dense in $(0,2 \pi)$. The result is assured by continuity of the correspondence (3.1).

Proof of Theorem 1.1. We construct a sequence $a_{n}$ that converges to $a \in\left(-\infty,-\frac{1}{4}\right)$ for which $\boldsymbol{K}\left(a_{n} \mid 1\right)$ converges. By Lemma 3.1, we may choose a sequence $\alpha_{1}, \alpha_{2}, \ldots$ in $\left(-\infty,-\frac{1}{4}\right)$ that converges to $a$ for which each map $s_{n}(z)=\alpha_{n} /(1+z)$ is of finite order. Let $\epsilon_{1}, \epsilon_{2}, \ldots$ be a sequence in $(0,1)$ that converges to 0 for which $\sum \epsilon_{n}$ diverges. Define $t_{n}(z)=\left(1-\epsilon_{n}\right) \alpha_{n} /(1+z)$, for $n=1,2, \ldots$ One may easily verify that

$$
t_{n} s_{n}^{-2} t_{n}(z)=z+\epsilon_{n}
$$


Since $s_{n}$ is of finite order, the two equal quantities in (3.2) are also equal to the $m$-fold composition $t_{n} s_{n} \cdots s_{n} t_{n}(z)$, where $m=\operatorname{order}\left(s_{n}\right)$.

For each $n$, choose an integer $N_{n}$ such that $N_{n} \epsilon_{n}$ is greater than the maximum element from the finite set

$$
\left\{\left|t_{n+1} s_{n+1}^{q}(0)\right|: 0 \leqslant q \leqslant \operatorname{order}\left(s_{n+1}\right)-2, t_{n+1} s_{n+1}^{q}(0) \neq \infty\right\} .
$$

Let $\phi_{n}$ represent the string of maps $t_{n}, s_{n}, \ldots, s_{n}, t_{n}$, in which $s_{n} \operatorname{occurs} \operatorname{order}\left(s_{n}\right)-2$ times. The continued fraction corresponding to the sequence of Möbius maps

$$
\phi_{1}, \ldots, \phi_{1}, \phi_{2}, \ldots, \phi_{2}, \ldots
$$

is the example we require, where the string $\phi_{n}$ occurs in the continued fraction $N_{n}$ times. To see that (3.4) provides an example of the required form, notice that the coefficients $a_{n}$ arise from the maps $s_{n}$ or $t_{n}$, thus certainly $a_{n} \rightarrow a$ as $n \rightarrow \infty$. It remains to demonstrate that the continued fraction converges (to $\infty$ ). This is true as

$$
\left(t_{1} s_{1}^{-2} t_{1}\right)^{N_{1}} \cdots\left(t_{n} s_{n}^{-2} t_{n}\right)^{N_{n}}(z)=z+\sum_{i=1}^{n} N_{i} \epsilon_{i},
$$

by (3.2), hence

$$
\begin{aligned}
\left(t_{1} s_{1}^{-2} t_{1}\right)^{N_{1}} \cdots\left(t_{n} s_{n}^{-2} t_{n}\right)^{N_{n}}\left(t_{n+1} s_{n+1}^{-2} t_{n+1}\right)^{p} t_{n+1} s_{n+1}^{q}(0) & \\
& =t_{n+1} s_{n+1}^{q}(0)+p \epsilon_{n+1}+\sum_{i=1}^{n} N_{i} \epsilon_{i} \\
& >\sum_{i=1}^{n-1} N_{i} \epsilon_{i},
\end{aligned}
$$

by (3.3), where $0 \leqslant p<N_{n+1}$ and $0 \leqslant q \leqslant \operatorname{order}\left(s_{n+1}\right)-2$. Therefore, the continued fraction converges to $\infty$.

Acknowledgements. This work was supported by Science Foundation Ireland Grant no. 05/RFP/MAT003.

\section{References}

1. A. F. BEARdon, The geometry of discrete groups (Springer, 1983).

2. A. F. BeARdon, Continued fractions, discrete groups and complex dynamics, Computat. Meth. Funct. Theory 1 (2001), 535-594.

3. A. A. Glutsyuk, On convergence of generalized continued fractions and Ramanujan's conjecture, C. R. Acad. Sci. Paris Sér. I 341 (2005), 427-432.

4. L. Jacobsen, R. L. Lamphere, G. E. Andrews and B. C. Berndt, The continued fractions found in the unorganized portions of Ramanujan's notebooks, Memoirs of the American Mathematical Society, Volume 99, No. 477 (American Mathematical Society, Providence, RI, 1992).

5. G. Jones AND D. Singerman, Complex functions: an algebraic and geometric viewpoint (Cambridge University Press, 1987).

6. M. Mandell and A. Magnus, On convergence of sequences of linear fractional transformations, Math. Z. 115(1) (1970), 11-17. 